\title{
Temperature and curvature-dependent thermal interface conductance between nanoscale-gold and water from molecular simulation
}

Blake A. Wilson ${ }^{1}$, Steven O. Nielsen ${ }^{2}$, Jaona H. Randrianalisoa ${ }^{3}$, Zhenpeng Qin ${ }^{1,4,5,6, *}$,

1 Department of Mechanical Engineering, The University of Texas at Dallas, Richardson, TX 75080, USA

2 Department of Chemistry and Biochemistry, The University of Texas at Dallas, Richardson, TX 75080, USA

3 Institut de Thermique, Mécanique, Matériaux EA 7548, University of Reims Champagne-Ardenne, Reims, France

4 Department of Bioengineering, The University of Texas at Dallas, Richardson, TX 75080, USA

5 The Center for Advanced Pain Studies, The University of Texas at Dallas, Richardson, TX 75080, USA

6 Department of Surgery, The University of Texas Southwestern Medical Center, Dallas, TX 75390, USA

* Zhenpeng.Qin@utdallas.edu

\begin{abstract}
Plasmonic gold nanoparticles (AuNPs) can convert laser irradiation into thermal energy and act as nano heaters in a variety of applications. Although the AuNP-water interface is an essential part of the plasmonic heating process, there is a lack of mechanistic understanding of how interface curvature and the heating itself impact interfacial heat transfer. Here, we report atomistic molecular dynamics simulations that investigate heat transfer through nanoscale gold-water interfaces. We confirmed that interfacial heat transfer is an important part of AuNP heat dissipation in AuNPs with diameter less than $100 \mathrm{~nm}$, particularly for small particles with diameter $\leq 10 \mathrm{~nm}$. To account for variations in the gold-water interaction strength reported in the literature, and to implicitly account for different surface functionalizations, we modeled a moderate and a poor AuNP-water wetting scenario. We found that the thermal interface conductance increases linearly with interface curvature regardless of the gold wettability, while it increases non-linearly, or remains constant, with the applied heat flux under different wetting conditions. Our analysis suggests the curvature dependence of the interface conductance is due to the changes in interfacial water adsorption, while the temperature dependence is caused by heat-induced shifts in the distribution of water vibrational states. Our study advances the current understanding of interface thermal conductance for a broad range of applications.
\end{abstract}

\section{Introduction}

Plasmonic nanoparticles can absorb and convert light into heat due to a photothermal process known as plasmonic heating. 1] In particular, gold nanoparticles (AuNPs) are commonly used as plasmonic nano heaters in a wide range of applications including hyperthermia-based tumor ablation, 2[5] selective protein inactivation, 6 - 10] nanobubble theranostics, 11] nanothermometry, 12 13] photothermal imaging, 14 15] and photoacoustic imaging. 16 17. Interfacial heat transfer is particularly relevant for nanoscale heat transfer because individual nanostructures exhibit a high surface-to-volume ratio and their ensembles have a high density of interfaces. 18 19] As such, heat transfer through the interface between a AuNP and its surrounding medium is an important element of the plasmonic heat dissipation process. 20 21] A thorough understanding of AuNP thermal interface conductance can be critical for precise control of plasmonic heat generation and its applications. 11 16 
Interfacial heat transfer can be characterized by the thermal interface conductance $(G)$, which quantifies the rate at which heat flows through an interface between two adjacent media at different temperatures. Experimental studies indicate that the thermal interface conductance of nanoparticles is affected by the chemical composition of the nanoparticle, its ligand coating, and the surrounding medium. $20|22| 26$ Molecular dynamics simulation studies of solid nanospheres immersed in mono-atomic fluids and nanodroplets of n-decane in water suggest that interface curvature (inversely proportional to particle size) and temperature can also affect nanoparticle thermal interface conductance. 27-29 Recent molecular dynamics studies also report that AuNPs immersed in organic solvent exhibit curvature dependent changes in thermal interface conductance. 30 31] Although previous experiments indicate that characteristic times for AuNP cooling in water scale with particle surface area, 32 it remains unclear if interface curvature or temperature affect the thermal conductance at nanoscale gold-water interfaces. These factors are of practical importance in biomedical applications that utilize plasmonic heating of AuNPs because the AuNPs are embedded in primarily aqueous media and may experience significant temperature variations.

In this article, we use atomistic molecular dynamics simulations to investigate the effects of temperature and curvature on heat transfer through bare nanoscale-gold interfaces with water during continuous plasmonic heating. Molecular dynamics simulations allow us to assess trends in the thermal interface conductance that can be difficult to explore experimentally due to limits in measurement sensitivity and particle monodispersity. 33. We simulated four nanoscale-gold surfaces with different curvature under two wetting conditions (moderate vs poor), and subjected each gold structure to various heating intensities to account for the effects of temperature increases during plasmonic heating. Altering the gold wettability allowed us to account for how differences in the gold-water interaction potential could affect the heat transfer, while also approximating the effects of coating the gold surface with ligands of different hydrophilicity. First, we confirmed that our results for the interface thermal conductance fall within the range of values reported from experiments. By comparing our results along with those from experiments to the critical value of the interface thermal conductance, we confirmed that the thermal interface conductance is an important element of AuNP heat dissipation, particularly, for small AuNPs $(\leq 10 \mathrm{~nm}$ in diameter $)$. Next, we analyzed the heat flux dependence and curvature dependence of the interface thermal conductance at the two wetting conditions. Our analysis indicates that the thermal interface conductance increases with curvature under both wetting conditions, but it increases non-linearly with heat flux only under moderate wetting of the gold surface. We found that the curvature dependence of the interface conductance coincided with a curvature dependent increase in water adsorption at the gold surfaces, consistent with trends observed previously for a simpler model of solid nanospheres immersed in monoatomic fluid. 27. We also observed a temperature dependent shift in the water vibrational density of states that improved the VDOS overlap with that of gold. This improved vibrational overlap may account for temperature dependent changes in the interface thermal conductance under the moderate wetting condition. This work advances our understanding of how temperature and curvature affect the thermal conductance across nanoscale gold-water interfaces.

\section{Results and Discussion}

\subsection{Analysis of the thermal interface conductance}

In order to evaluate the impact of interface curvature on the heat transfer between nanoscale-gold and water, we modeled in atomistic detail four nanoscale gold-water interfaces of different curvatures (Figure 1a): three spherical AuNPs with diameters $(d)$ of 3, 5 , and $10 \mathrm{~nm}$, as well as a planar gold-water interface to represent the zero curvature limit. To estimate the thermal interface conductance we performed steady-state non-equilibrium molecular dynamics simulations in which the gold structure was subjected to a constant heat power, $Q$, while an outer region of water was maintained at $300 \mathrm{~K}$ as a heat sink (Figure 1b), approximating plasmonic heating of gold under continuous-wave laser stimulation. Under these conditions, the steady-state temperature profile in the direction normal to the interface between gold and water exhibits a temperature jump $(\Delta T)$ (Figure 1c), which is inversely proportional to the thermal interface conductance, $G$,

$$
G=\frac{Q}{A \Delta T}=\frac{q}{\Delta T}
$$


(a)

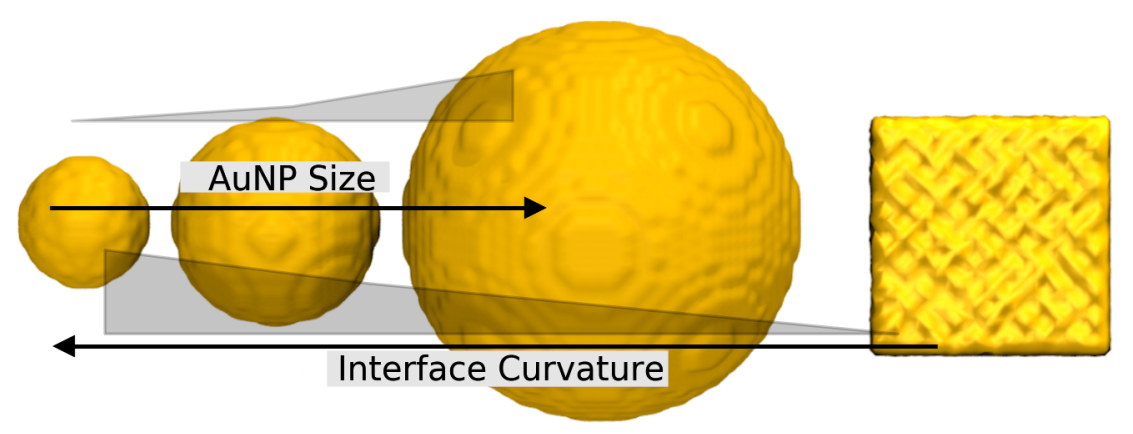
$3 \mathbf{n m}$
$5 \mathbf{n m}$
$10 \mathrm{~nm}$
planar

(b)

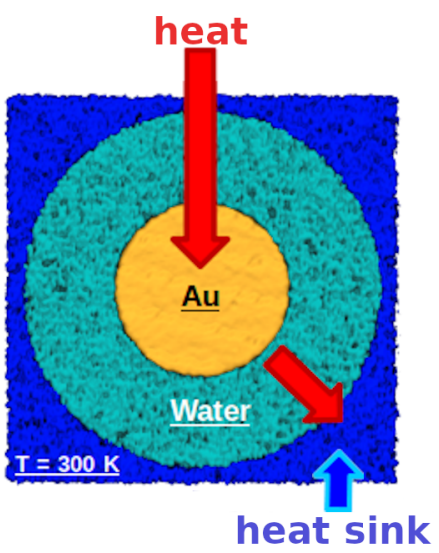

(c)

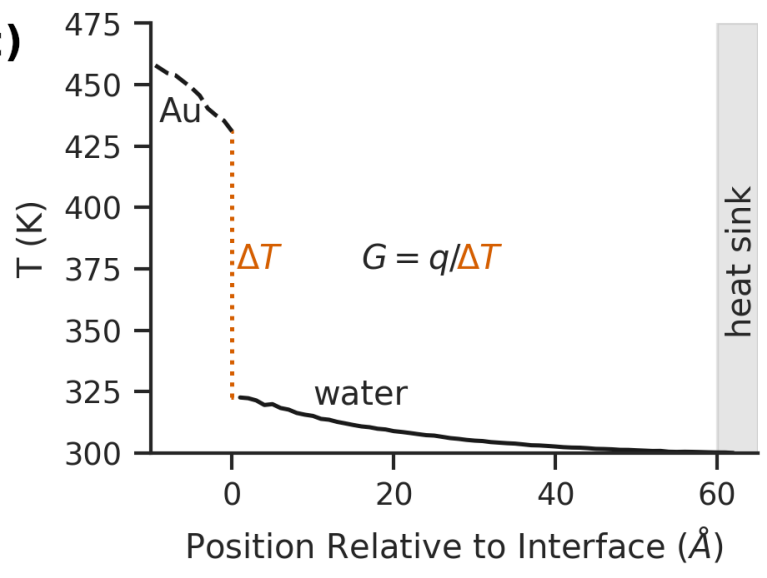

Figure 1. Simulation design. a) Images depicting the simulated gold surfaces. b) Simulation design for the spherical AuNP geometry. Heat is applied uniformly to the gold atoms while the dark blue region of water acts as a heat sink, maintained at $\mathrm{T}=300 \mathrm{~K}$. c) Representative temperature profile for a AuNP under continuous heating, which demonstrates the temperature jump at the interface. 
where $q=Q / A$ is the heat flux across the interfacial area $A$, which is taken as the gold surface area: $A=\pi d^{2}$ for spherical AuNPs with diameter $d$ and $A=2 L_{x} L_{y}$ for the planar gold surface. $L_{x}$ and $L_{y}$ are the lateral dimensions of the gold surface within the simulation cell, and the factor of two accounts for the fact that two sides of the planar surface are exposed to solvent. To consider the effects of temperature, we conducted simulations under various heating powers $(Q$, Table S1, Supporting Information). Note that in some cases, the heating caused the AuNPs to thermally expand (Table S2, Supporting Information), which was accounted for when computing the thermal interface conductance.

$G$ is strongly affected by solvent adsorption (i.e., wetting) at the interface, scaling with the strength of the interaction between the solid and liquid atoms. $27[34$ To account for how differences in wetting at the gold-water interface may affect the curvature and temperature dependence of $G$, we modeled two wetting conditions: a moderate wetting case using the gold-water interaction parameters developed by Merabia et al. to reproduce a gold-water contact angle $<30^{\circ}, 35$ as well as a poor wetting case in which we reduced the gold wettability by decreasing the strength of the gold-water interaction by roughly a factor of 4 . There are three reasons to consider multiple wetting conditions for the gold-water interface. First, there are various parameterizations of the gold-water interaction available in the literature with different parameter values and energy functions, 36 39] the particular choice of which can affect both the wetting and thermal interface conductance. 40 Second, the standard 12-6 Lennard-Jones potential we used to model the gold-water interaction (Equation 5) does not explicitly include image charge effects arising from polarization of the metal atoms at an interface, 4142 which can also affect adsorption at the metal surface. Polarization can also affect the thermal interface conductance. 43 Lastly, the hydrophilicity of solid-water interfaces can be tuned by ligand functionalization, 44 with hydrophobic ligands yielding lower thermal interface conductance than hydrophilic ones. $25[45]$ Altering the gold wettability approximates the effect of coating the gold surface with ligands of varying hydrophilicity.

Our simulations fall within the previous measured range for $G$. The steady-state temperature profiles of gold and water for each case are shown in Figure S1 and S2 (Supporting Information). The thermal interface conductance $(G)$ was determined according to Equation 1. $G$ ranged from $\sim 110-185 \mathrm{MW} \mathrm{m}^{-2} \mathrm{~K}^{-1}$ for the moderate wetting case and $\sim 20-45 \mathrm{MW} \mathrm{m}^{-2} \mathrm{~K}^{-1}$ for the poor wetting case. The AuNP results under moderate wetting fall within the range of experimental results $\left(\sim 100-250 \mathrm{MW} \mathrm{m}^{-2} \mathrm{~K}^{-1}\right)$ reported previously for various spherical nanoparticle-water interfaces (Figure 2), $20.22[26$ while the poor wetting results fall within the range of values $(G \sim 5-50$

$\mathrm{MW} \mathrm{m}^{-2} \mathrm{~K}^{-1}$ ) reported from experiments of spherical AuNPs in organic solvents. 202226 values for CTAB coated gold nanorods in water have also been reported, with estimates ranging from $\sim 50-450 \mathrm{MW} \mathrm{m}^{-2} \mathrm{~K}^{-1}$. 24$] 48$. $G$ of the planar surface in the moderate wetting case was $\sim 110 \mathrm{MW} \mathrm{m}^{-2} \mathrm{~K}^{-1}$ at all heating powers, which is close to the previously reported values of $105 \pm 15 \mathrm{MW} \mathrm{m}^{-2} \mathrm{~K}^{-1}$ for $100 \mathrm{~nm}$ and $110 \pm 10 \mathrm{MW} \mathrm{m}^{-2} \mathrm{~K}^{-1}$ for $18.5 \pm 3.0 \mathrm{~nm}$ citrate-stabilized AuNPs in water. 21 26 The planar surface had a $G \sim 25-26 \mathrm{MW} \mathrm{m}^{-2} \mathrm{~K}^{-1}$ in the poor wetting case.

\subsubsection{Importance of thermal interface conductance for nanoparticles}

For spherical AuNPs the relative importance of $G$ in regulating their heat dissipation can be gauged by the Kapitza number, 1$]$

$$
\lambda_{K}=\frac{2 l_{K}}{d}=\frac{2 \kappa_{W}}{G d},
$$

where $l_{K}$ is the Kapitza length, 4950$]$ is the particle diameter, and $\kappa_{W}$ is the thermal conductivity of the surrounding water. If $\lambda_{K} \ll 1$, the heat transport is limited by heat diffusion through water and interfacial heat transfer has little effect on the process. In contrast, if $\lambda_{K} \gg 1$ the heat transport is limited by the interface. By setting $\lambda_{K}=1$, we can define a critical $G$ value,

$$
G_{c}=\frac{2 \kappa_{W}}{d}
$$

Analogously, when $G \gg G_{c}$ AuNP heat dissipation is limited by heat diffusion through water and when $G \ll G_{c}$ it is limited by the interface. Heat transfer through the interface only has a negligible impact on the kinetics of AuNP 


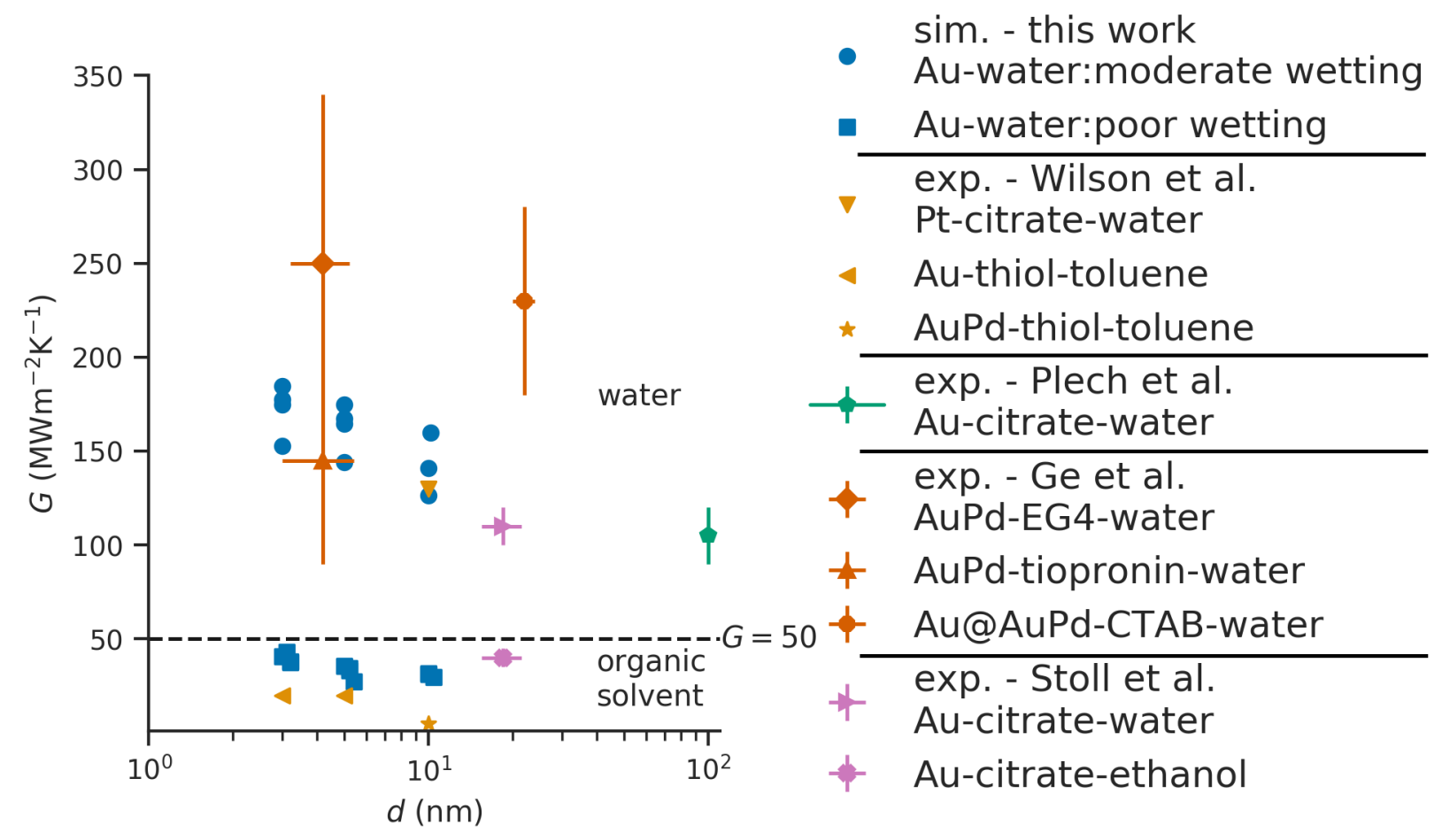

Figure 2. Thermal interface conductance. The simulated nanoscale gold-water thermal interface conductance values (this study) are plotted versus particle diameter, along with experimental data for spherical nanoparticles in water. Note that the different values of $G$ shown for the simulated AuNPs at a given size correspond to simulations at different heating power. Experimental data were collected from Wilson et al., 20 Plech et al., 21 Ge et al., 22 and Stoll et al. 26. Error bars were included for both $G$ and $d$ of the experimental data points if specified in the given references. Note that we used the lower limit value of $20 \mathrm{MW} \mathrm{m}^{-2} \mathrm{~K}^{-1}$ reported by Wilson et al. in the plot for the Au-thiol-toluene interface. 20 
heat dissipation in the former case (i.e., when $G \gg G_{c}$ ). To gauge the relative importance of heat transfer through the gold-water interface to AuNP heat dissipation, we compared the $G$ values from our simulated cases as well as those reported from experiments of AuNPs in water with $G_{c}$ evaluated across a range of AuNP sizes (Figure 3). From this analysis, we confirm that for AuNPs within the size range we have simulated $(d \leq 10 \mathrm{~nm})$ interfacial heat

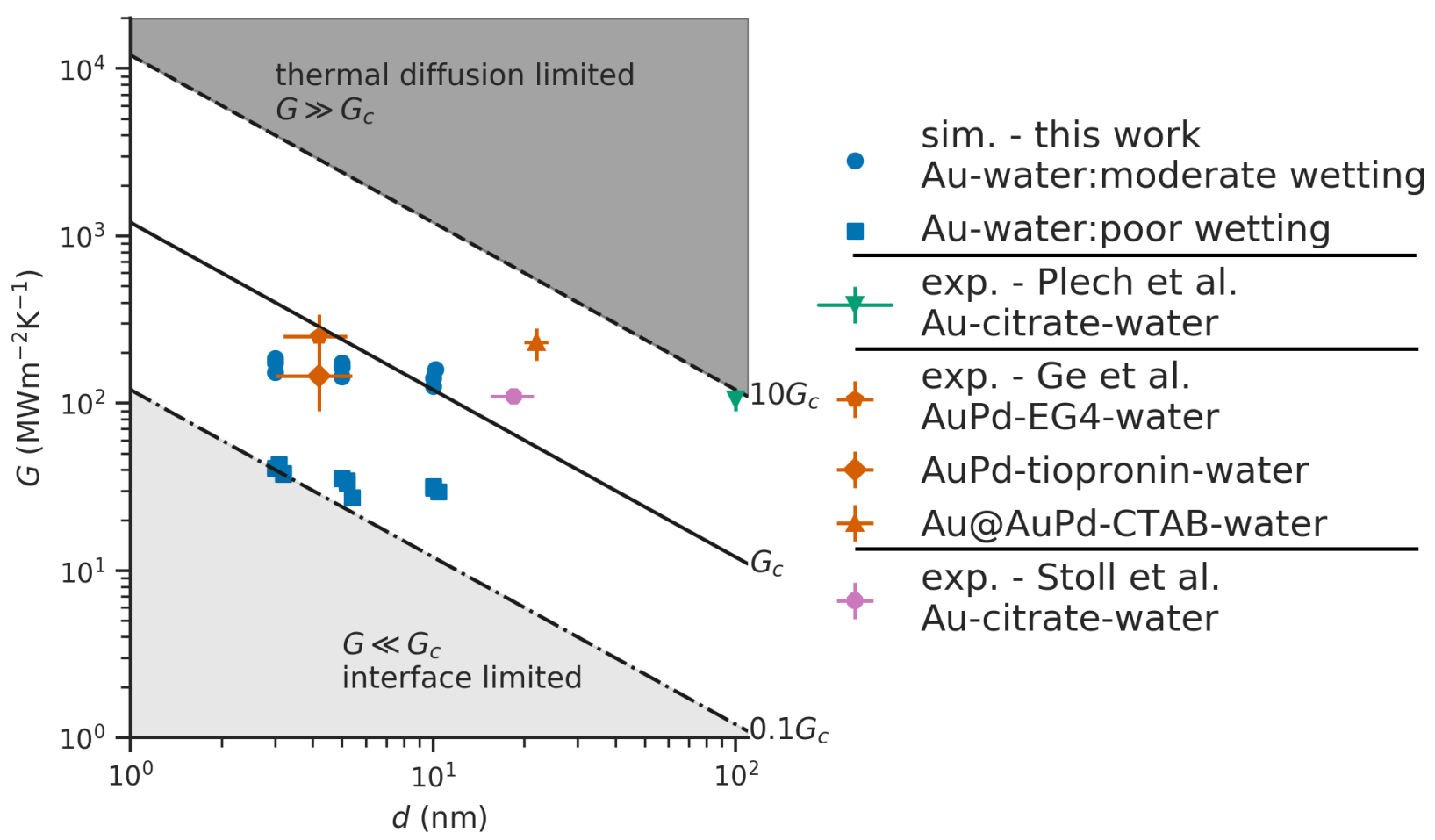

Figure 3. Critical thermal interface conductance. The simulated nanoscale gold-water thermal interface conductance values (this study) are plotted versus particle diameter and compared to the critical value of the thermal interface conductance $G_{c}$ (solid line), along with experimental data for spherical AuNPs in water. Experimental data were collected from Plech et al., 21] Ge et al., 22] and Stoll et al. 26 The boundaries between the thermal diffusion limited regime (dark gray region) and the interface limited regime (light gray region) were taken as $G=10 G_{c} \gg G_{c}$ (dashed line) and $G=0.1 G_{c} \ll G_{c}$ (dash-dot line), respectively. A constant $\kappa_{W}=0.6 \mathrm{~W} \mathrm{~m}^{-1} \mathrm{~K}^{-1}$ was used when computing $G_{c}$ values.

transport is a particularly important factor in their heat dissipation, with $G \lesssim G_{c}$ under both wetting conditions. For the simulated poor wetting case, the $G$ values of the 3 and $5 \mathrm{~nm}$ AuNPs even approach the boundary for interface limited heat dissipation. Considering the experimental estimates of $G$ for AuNPs in water, 21 22 26] it appears that $G$ only approaches the boundary for thermal diffusion limited heat dissipation around $d \sim 100 \mathrm{~nm}$, suggesting that the interface slows AuNP heat dissipation for diameters $d<100 \mathrm{~nm}$. However, coating AuNPs with a sufficiently hydrophilic ligand such as PEG may lead to much higher $G$, 25. which may make heat dissipation out of particles with $d<100 \mathrm{~nm}$ thermal diffusion limited. Conversely, $G$ for CTAB stabilized gold nanorods in water may be as low as $\sim 50 \mathrm{MW} \mathrm{m}^{-2} \mathrm{~K}^{-1}$ in some cases, 48 so more hydrophobic ligands will promote interface limited heat dissipation out of AuNPs with $d \leq 5 \mathrm{~nm}$.

\subsubsection{Temperature dependence}

For the moderate wetting case, $G$ of the spherical AuNPs exhibited a non-linear dependence on the heat flux

(Figure 4a), increasing by about 20-40\% from the lowest to the highest heating intensities for the spherical AuNPs. Since the applied heating power affects the temperature of both the gold structure and surrounding water (Figure S3, 

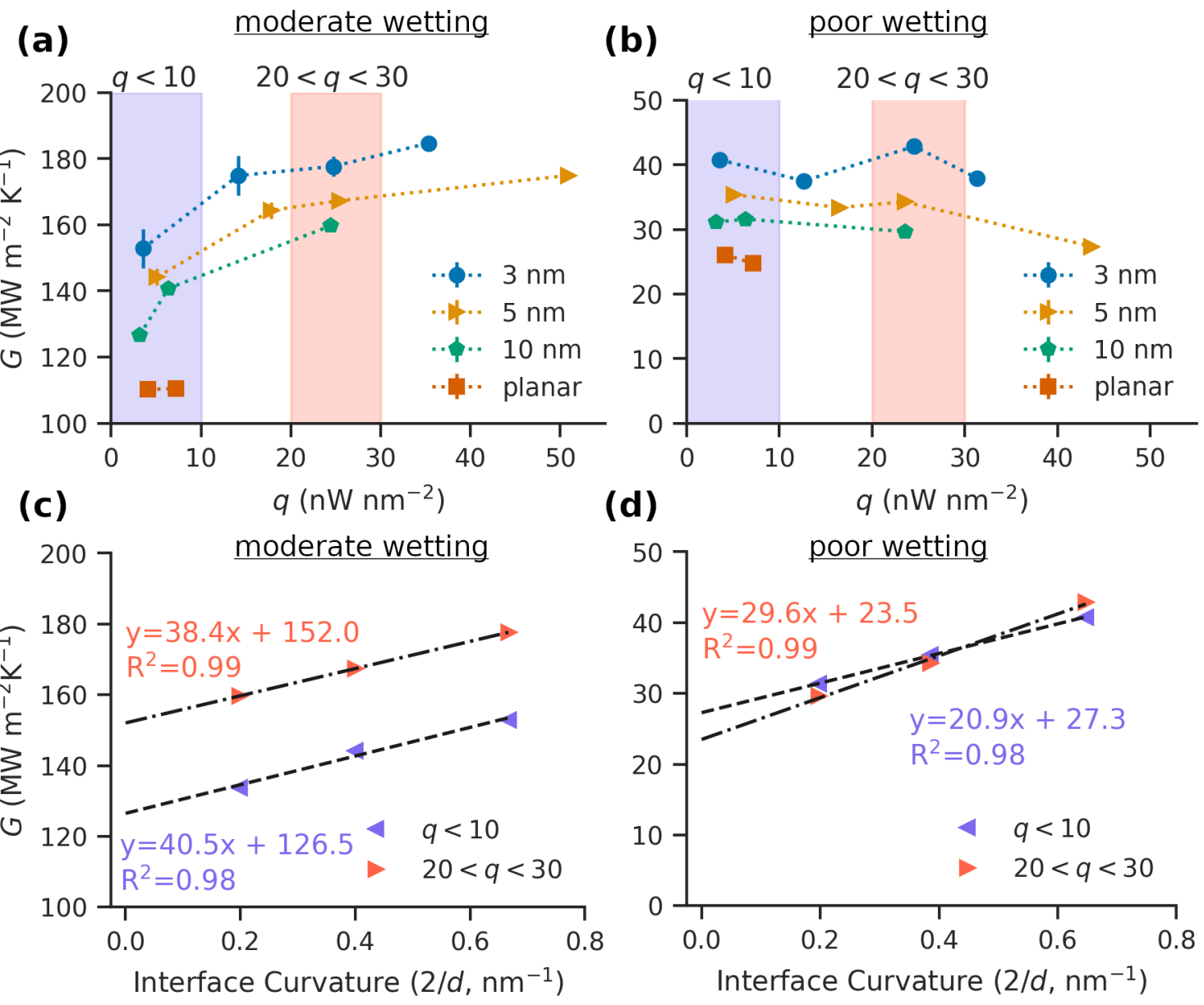

Figure 4. a) Thermal interface conductance versus heat flux for the different gold-water interfaces in the moderate wetting case. b) Thermal interface conductance versus heat flux for the different gold-water interfaces in the poor wetting case. c) Interface conductance versus interface curvature under different levels of heating for the moderate wetting case. d) Interface conductance versus heat flux for different gold-water interfaces for the poor wetting case. The dashed and dash-dot lines in (c) and (d) are the linear-fit lines consistent with Equation 4 . y $\left(\mathrm{MW} \mathrm{m}^{-2} \mathrm{~K}^{-1}\right)$ corresponds to $G(d), x\left(\mathrm{~nm}^{-1}\right)$ corresponds to $2 / d$, the slope $\left(\times 1000^{-1} \mathrm{MW} \mathrm{m}^{-1} \mathrm{~K}^{-1}\right)$ corresponds to $\delta$, and the y-intercept $\left(\mathrm{MW} \mathrm{m}^{-2} \mathrm{~K}^{-1}\right)$ is $G_{0}$. 
Supporting Information), an increase in the thermal interface conductance with heat flux suggests an underlying

Temperature dependent thermal conductance has been observed in other systems. A linear dependence on temperature has been observed for thermal conductance at solid-solid interfaces at high temperature, which was attributed to an increase in inelastic scattering of phonons at the interface. 51 52 Various simulation studies have reported that the thermal interface conductance of nanoscale solid-fluid interfaces tends to increase with temperature, 53 55. typically following a power law expression. However, others indicate a decrease in the thermal interface conductance with temperature. $28|35| 56 \mid 57$ Chen et al. reported a dependence of the thermal interface conductance on heat intensity for simulations of a $3 \mathrm{~nm}$ spherical AuNP immersed in a flexible water model that was similar to the one we observed for the AuNPs, 40 but they also found that the thermal interface conductance was higher overall and did not change with heating power with a rigid water model. 40

\subsubsection{Curvature dependence}

Under both wetting conditions, the relative values of $G$ are in order of planar $<10 \mathrm{~nm} \mathrm{AuNP}<5 \mathrm{~nm} \mathrm{AuNP}<3 \mathrm{~nm}$ AuNP (Figure 4a,b), suggesting a dependence on the interface curvature. Tascini et al. reported that the thermal interface conductance of solid nanospheres immersed in a monoatomic fluid increased linearly with interface curvature according to the following expression: 27

$$
G(d)=\delta \frac{2}{d}+G_{0}
$$

where $d$ is particle diameter and $2 / d$ the interface curvature, $\delta$ is the change in interface conductance with curvature, and $G_{0}$ is the interface conductance at the zero-curvature limit (i.e., $\lim _{2 / d \rightarrow 0}$ ) which should match the conductance of the corresponding planar surface. Similarly, we found that at low and high heat flux the thermal interface conductance of the spherical AuNPs increased linearly with the interface curvature in each heating regime as per Equation 4 (Figure 4c,d); note that for both wetting conditions we averaged the two $G$ values for the $10 \mathrm{~nm}$ AuNP in the low heat flux regime for the linear fit. For the moderate wetting case, the $\delta$ values were similar in both heating regimes $\left(\sim 0.04 \mathrm{~W} \mathrm{~m}^{-1} \mathrm{~K}^{-1}\right)$, and were consistent with values reported by Tascini et al. for nanoparticles with full wetting $\left(\delta \sim 0.023-0.105 \mathrm{~W} \mathrm{~m}^{-1} \mathrm{~K}^{-1}\right)$. 27 However, the corresponding values of $G_{0}$ were different due to the increase in $G$ with heat flux. $G_{0}$ for the fit to the AuNP data in the low-heat flux regime $\left(126.5 \mathrm{MW} \mathrm{m}^{-2} \mathrm{~K}^{-1}\right)$ was also higher than the thermal interface conductance of the planar gold interface $\left(\sim 110 \mathrm{MW} \mathrm{m}^{-2} \mathrm{~K}^{-1}\right)$ in that heat flux regime. However, given that the heat fluxes aren't exactly the same across the interfaces and that we averaged the two $G$ values for the $10 \mathrm{~nm}$ AuNP in the low heat flux regime, as well as the limited number of AuNP sizes available for the linear fit, some error in the estimate of $G_{0}$ is to be expected. In the poor wetting case, the $\delta$ values in the two heating regimes $\left(\sim 0.021\right.$ and $\left.\sim 0.03 \mathrm{~W} \mathrm{~m}^{-1} \mathrm{~K}^{-1}\right)$ were lower than in the moderate wetting case, consistent with values reported by Tascini et al. for nanoparticles with partial $\left(\delta \sim 0.012-0.023 \mathrm{~W} \mathrm{~m}^{-1} \mathrm{~K}^{-1}\right)$ and full wetting $\left(\delta \sim 0.023-0.105 \mathrm{~W} \mathrm{~m}^{-1} \mathrm{~K}^{-1}\right) .27$ In this case, the planar surface $G$ values $\sim 25-26 \mathrm{MW} \mathrm{m}^{-2} \mathrm{~K}^{-1}$ fall with the range of the $G_{0}$ estimates (27.3 and $23.5 \mathrm{MW} \mathrm{m}^{-2} \mathrm{~K}^{-1}$ for low and high heat flux, respectively).

Curvature dependence of the interface thermal conductance has previously been reported from simulations of other systems including simpler solid nanospheres in mono-atomic Lennard-Jones fluids, 27 [28] and nanodroplets of n-decane in water, 29. as well as solid and liquid $\mathrm{ZnO}$ nanoparticles in tetradecane. 58 In those cases, as with our simulations of the gold-water interface, $\delta>0$ and the interface conductance increased with curvature (decreases with particle size) (Table S3, Supporting Information). In contrast, $G$ from simulations of bare spherical AuNPs in hexane exhibited the opposite trend with $\delta<0$ ( $G$ decreases with curvature). 30 31] In this case, the curvature dependence was attributed to size dependent changes in the structure, coordination, and vibrational modes of the AuNP surface atoms. In the case of n-decane nanodroplets, the increase in $G$ with curvature (i.e., $\delta>0$ ) was attributed to a reduction in the nanodroplet-water interface tension, 29] while in the case of solid nanospheres in momo-atomic fluid Tascini et al. found that $\delta>0$ corresponded to curvature dependent differences in solvent adsorption density at the nanoparticle surfaces. 27 


\subsection{Analysis of water adsorption at the nanoscale-gold surfaces}

The structuring and adsorption of solvent at a solid surface is an important factor in the interfacial heat transfer and can have a large effect on thermal interface conductance. 34 45[59] For solid spherical nanoparticles, Tascini et al. found that smaller nanoparticles with larger curvature had higher surface adsorption density 27, which coincided with the increases in $G$ that they observed with the particle curvature. To determine if curvature dependent changes in solvent adsorption could explain the curvature dependence of the AuNP-water $G$, we examined the structuring and adsorption of water at the surfaces of nanoscale gold (Figure 5a). For each wetting condition, we first examined the equilibrium density profiles of gold and water at $300 \mathrm{~K}$ (i.e., no heating) (Figure 5b,c). In the moderate wetting case, the peak in the mass density corresponding to the adsorption layer was $1.7 \mathrm{~g} \mathrm{~mL}^{-1}$ for the AuNPs and $2.4 \mathrm{~g} \mathrm{~mL}^{-1}$ at the planar interface (Figure 5b), whereas in the poor wetting case it was $1.4 \mathrm{~g} \mathrm{~mL}^{-1}$ for all the gold structures (Figure 5c). In the absence of heating, the surface adsorption density, $\varrho$ (Equation 8 9), increased linearly with the gold-water interface curvature (Figure 6a), regardless of the wetting strength. This result is consistent with the trends reported by Tascini et al. who observed an increase in surface adsorption density with interface curvature in simulations of spherical NPs immersed in a monoatomic fluid at various wetting strengths. 27 To better understand the curvature dependence of water adsorption at the gold surfaces, we examined how the relative solvent-accessibility of the gold structures changed with interface curvature. Measures of solvent accessibility were developed in the context of proteins, 60 61] as a metric to help quantify the role of hydration in protein structure, folding and binding. We computed the solvent accessible surface area, $A_{\mathrm{SASA}}$ (see Section 4.3.5), of the initial configuration of each gold structure and normalized it relative to its surface area $\left(A=\pi d^{2}\right.$ for spherical AuNPs and $A=2 L_{x} L_{y}$ for the planar slab) to quantify the relative accessibility: $A_{\mathrm{SASA}} / A$. The relative accessibility $A_{\mathrm{SASA}} / A$ increased linearly with the interface curvature (Figure $6 \mathrm{~b}$ ). Since $A_{\mathrm{SASA}}$ effectively quantifies the maximum amount of water that can adhere at the gold surfaces independent of wetting strength and temperature/heating, the trend in $A_{\mathrm{SASA}} / A$ suggests a geometric effect in which there is more space for water to adsorb at the curved gold surfaces. This effect explains why the more highly curved gold surfaces sustain higher $\varrho$ in both the moderate and poor wetting conditions.

We also analyzed the steady-state non-equilibrium molecular dynamics simulations and evaluated the steady-state density profiles of gold and water under each simulated heating power (Figure S4 and S5, Supporting Information). In most cases, there were only minor changes in the height and width of the peak corresponding to the water adsorption layer with heating. However, in the poor wetting case the distinct peak for the adsorption layer near the 5 and $10 \mathrm{~nm}$ AuNPs disappeared at heating powers of $Q=4000 \mathrm{nW}$ and $Q=8000 \mathrm{nW}$, respectively (Figure S5, Supporting Information), exhibiting signs of temperature induced desorption. Regardless, the temperature of water at the interfaces was well below the threshold for vapor nanobubble formation (Figure S3b, Supporting Information). 11] The gold particles were however molten in those cases (Figure S3d). Putting aside the two cases that exhibited signs of temperature induced desorption, under both wetting conditions the surface adsorption density $\varrho$ was relatively unaffected by heating and did not exhibit any clear dependence on heat flux (Figure 6c,d). This result suggests that the amount of water in the first adsorption layer remains relatively constant during heating, and it is consistent with the temperature induced desorption being a threshold event that depends on both the water temperature and the gold wettability.

\subsection{Analysis of nanoscale-gold and water vibrational density of states}

In the absence of electron-phonon coupling, interfacial heat transport is mediated by the transmission and scattering of phonons (vibrational energy carriers) across the interface. $\frac{18 \mid 62}{62}$ In particular, models of thermal interface conductance based on diffuse mismatch emphasize coupling between the two materials' vibrational modes, or phonon density of states, as a critical factor in heat transport through the interface. 62 63 In molecular dynamics simulations, the vibrational coupling between nanomaterials and solvent can be qualitatively characterized by computing their respective vibrational density of states (VDOS) and comparing the overlap between the two distributions. 27 31 35 40 We therefore computed the VDOS (Equation 10 of each gold structure and the surrounding water at $300 \mathrm{~K}$ (Figure 7a). The water distribution was broad, exhibiting a low intensity peak in the low frequency domain and a broad peak at higher frequency, consistent with previous reports. 30.40 . Only the low frequency water peak has significant overlap with the VDOS of gold. The VDOS of the gold was concentrated in the low frequency regime with two distinct peaks similar to the tranverse (TA) and longitudinal (LA) acoustic modes in bulk gold, 64

162 163 164 165 166 167 168 169 170 171 172 


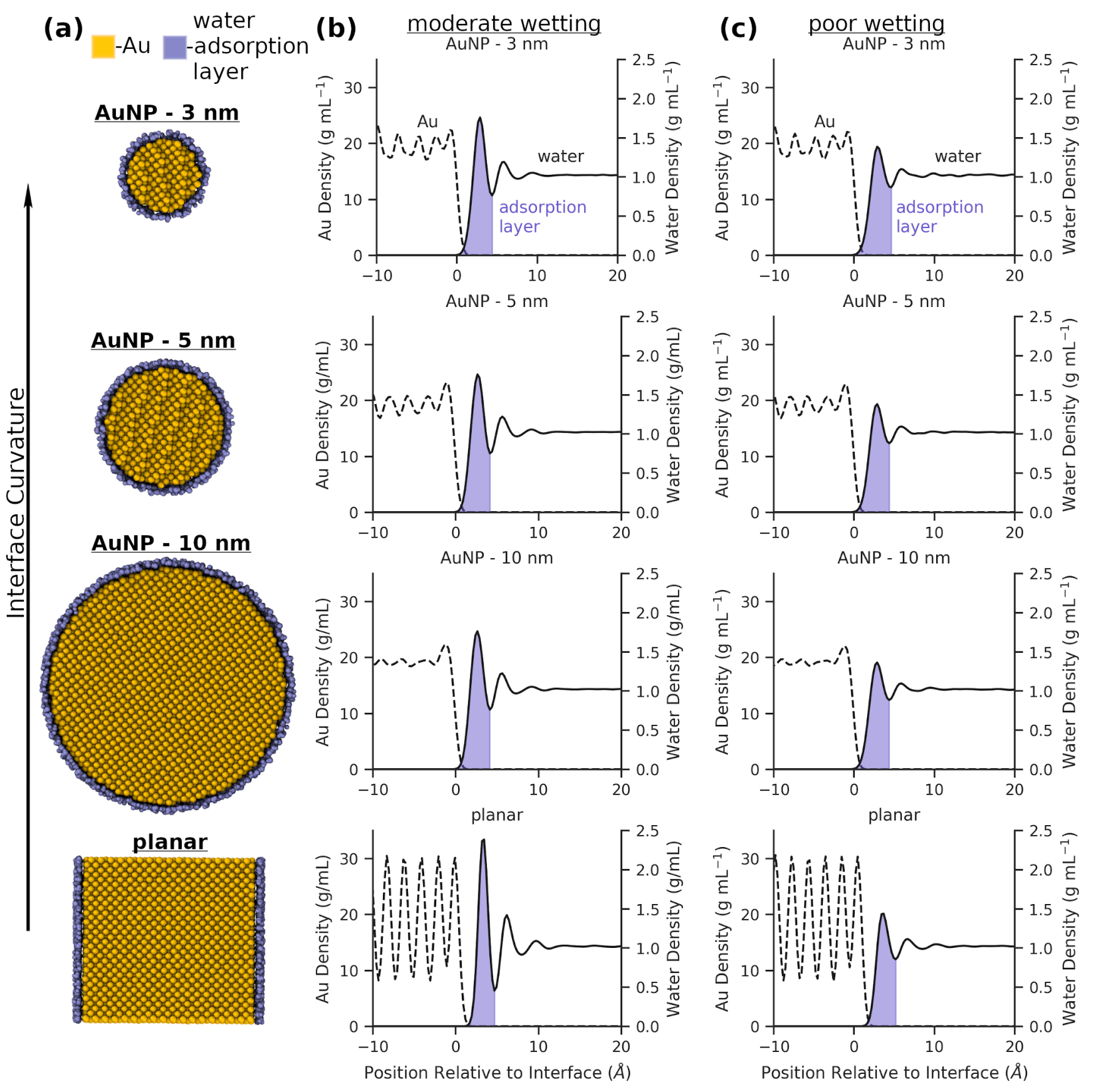

Figure 5. Water adsorption at the surface of nanoscale-gold a) Representative snapshot of a cross-section view of the AuNP-water interface (without heating, moderate wetting) showing the first layer of water adsorbed onto the gold surface. b,c) Mass density profiles of gold and water in the absence of heating ( $T=300 \mathrm{~K})$ for the moderate (b) and poor wetting (c) cases; the layer of water adsorbed onto the gold surface is shaded in purple. 

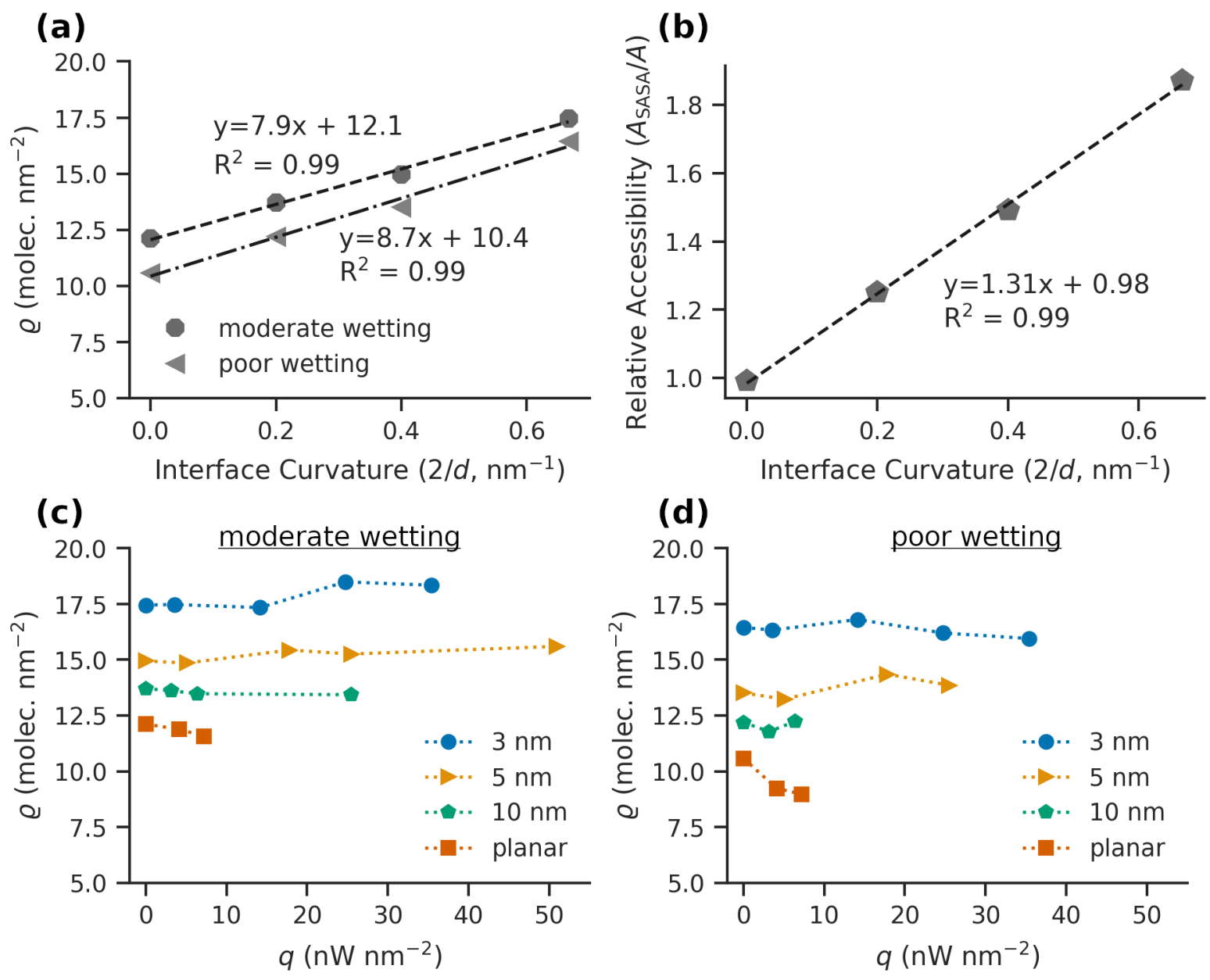

Figure 6. a) Surface adsorption density of water versus interface curvature in the absence of heating for the moderate and poor wetting cases; the dashed line shows the linear fit. b) Relative accessibility $\left(A_{\mathrm{SASA}} / A\right)$ versus interface curvature. The dashed line shows the linear fit to the data. c,d) Water adsorption density versus heat flux for the different gold-water interfaces under normal (c) and reduced (d) wetting conditions. 
consistent with previous reports (Figure 7a). $40[65$ Increasing the interface curvature leads to lower peak intensity and broader outer tails in the gold VDOS, especially for the LA mode (Figure 7a, inset). Since the temperature of gold and nearby water are elevated (above $300 \mathrm{~K}$ ) during heating (Figure S5, Supporting Information), we also computed the vibrational density of states at $370 \mathrm{~K}$ and compared the results to those at $300 \mathrm{~K}$. The $3 \mathrm{~nm}$ AuNP exhibited the most obvious shift in VDOS (Figure 7b), but overall the temperature dependent changes in the gold VDOS were minor (Figure $7 \mathrm{~b}$ and Figure S6, Supporting Information). In contrast, the water density underwent a noticeable redistribution to the lower frequency regime at $370 \mathrm{~K}$ (Figure $7 \mathrm{c}$ ). The percentage of the water VDOS in the range 0 to $6 \mathrm{THz}$ increased from $11 \%$ at $300 \mathrm{~K}$ to $14 \%$ at $370 \mathrm{~K}$, which suggests there may be better vibrational coupling between gold and water at elevated water temperatures. Therefore, temperature dependent shifts in the water VDOS may contribute to the increases in $G$ with heat flux observed in the moderate wetting case.

To further characterize the level of overlap between the gold and water VDOS we computed the Bhattacharyya coefficient (BC, Equation 11, 66 a measure of overlap between probability distributions which ranges from 0 (no overlap) to 1 (perfect overlap), between the VDOS of each gold structure and water (Figure $7 \mathrm{~d}$ ). At $300 \mathrm{~K}$, the $B C$ values ranged from $0.28-0.29$. At $370 \mathrm{~K}$, the $B C$ values increased to $0.30-0.32$, indicating improved overlap between the gold and water VDOS. To further confirm this effect, we computed the cross-temperature $B C$ values (Figure $7 \mathrm{e}$ ). Similarly, the $B C$ only increased when the water temperature was $370 \mathrm{~K}$, confirming that shifts in the water VDOS are the primary factor improving the overlap between the gold and water VDOS.

\section{Conclusions}

In this work, we used atomistic molecular dynamics simulations to investigate the heat transfer through nanoscale gold-water interfaces. We simulated four nanoscale-gold surfaces with differing curvature, subjected to various heating intensities, and under two wetting conditions. An analysis of the critical value of the thermal interface conductance confirmed that the thermal interface conductance is an important factor in the aqueous heat transfer of particles in the size range we investigated. We found that for a given AuNP size, the thermal interface conductance increased non-linearly with the applied heat flux under moderate wetting of the gold surface, and did not change under poor wetting conditions. Analysis of the gold and water vibrational density of states showed a temperature dependent shift in the water distribution that improved the overlap with the gold density of states. This shift in the water VDOS may contribute to the increases in thermal interface conductance with applied heat flux under the moderate wetting condition. Our results also showed that the thermal interface conductance between nanoscale-gold and water increased linearly with interface curvature at both low and high heat flux under both wetting conditions. Our analysis indicated that the curvature dependence of the interface conductance coincided with a curvature dependent increase in water adsorption at the gold surfaces, consistent with that observed previously with a simpler model of solid nanospheres immersed in monoatomic fluid. 27

This work helps elucidate the temperature and curvature effects on the thermal conductance across nanoscale gold-water interfaces. Our work here focused on bare spherical particles immersed in water. Although we considered two conditions for the gold wettability which approximates the effect of coating the gold with ligands of different hydrophilicity, there are additional effects that ligand functionalization can have on interfacial heat transfer that were not directly accounted for in our study. 30 46 47 We also did not directly investigate how the observed temperature and curvature dependent changes in the thermal interface conductance impact the spatiotemporal temperature dynamics during pulsed laser stimulation of AuNPs, or how they might affect any particular applications of photothermally heated AuNPs. Future work will be required to address these issues, as well as to experimentally validate our findings. 


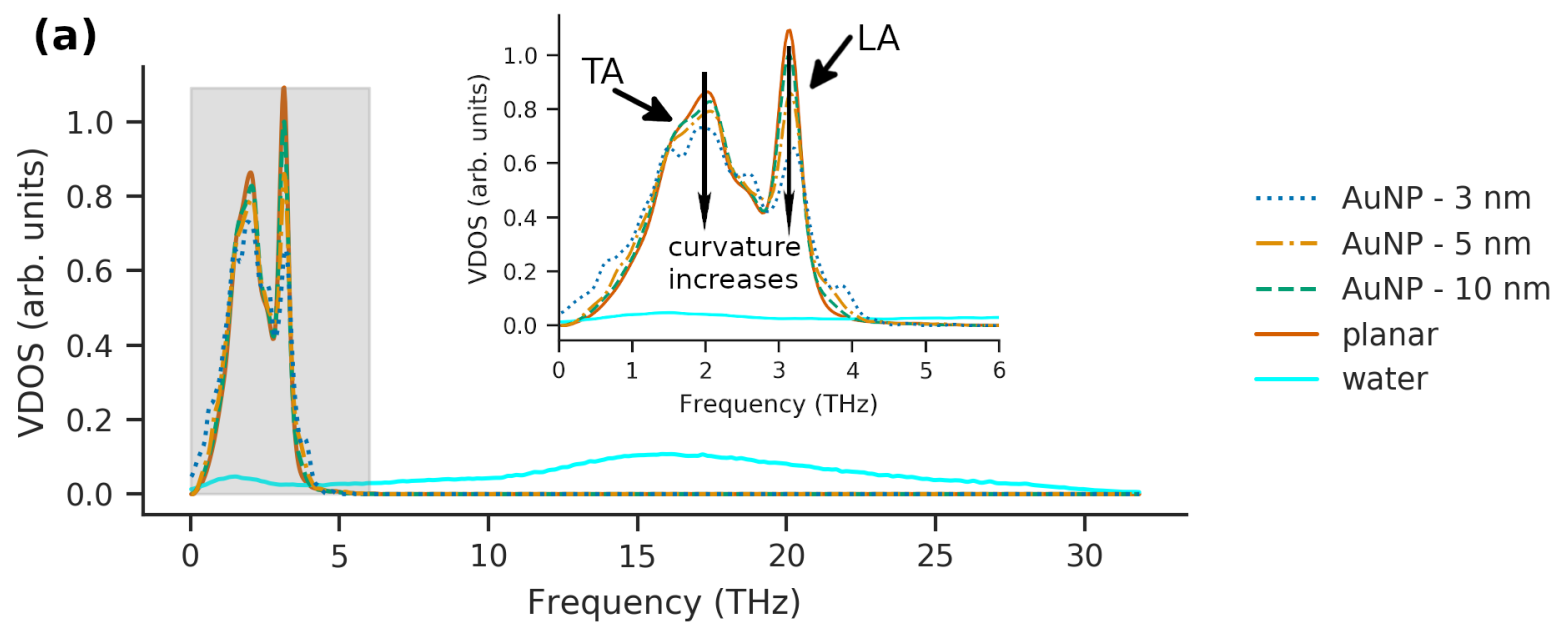

(b)

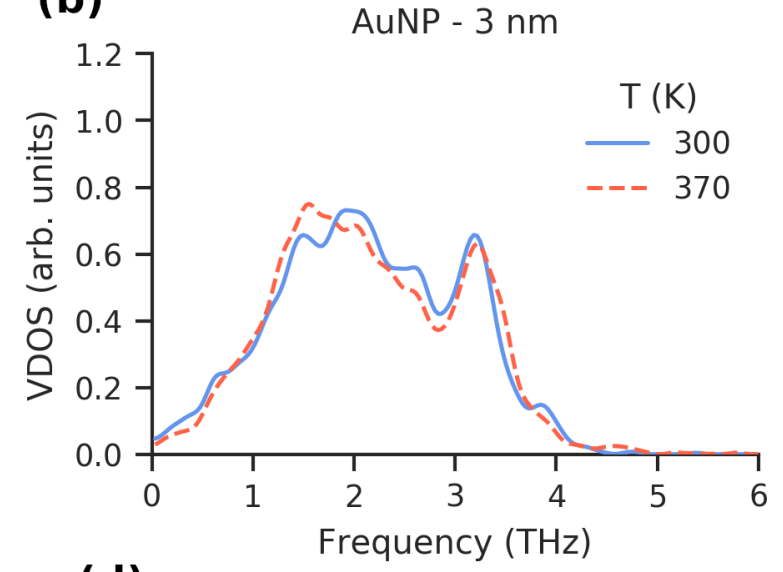

(d)

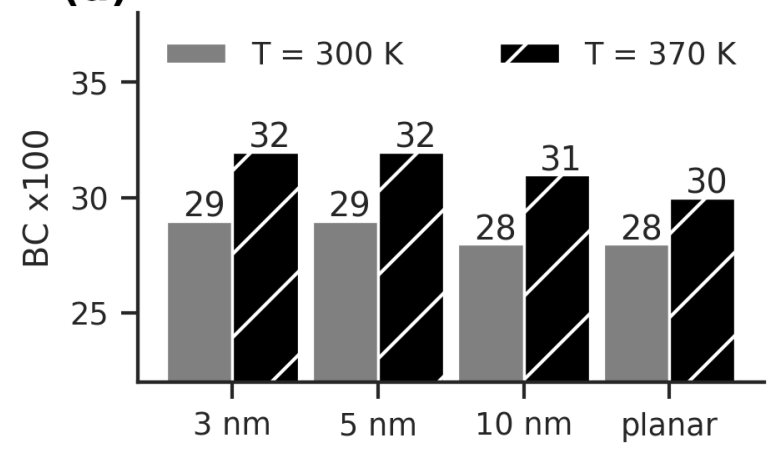

(c)

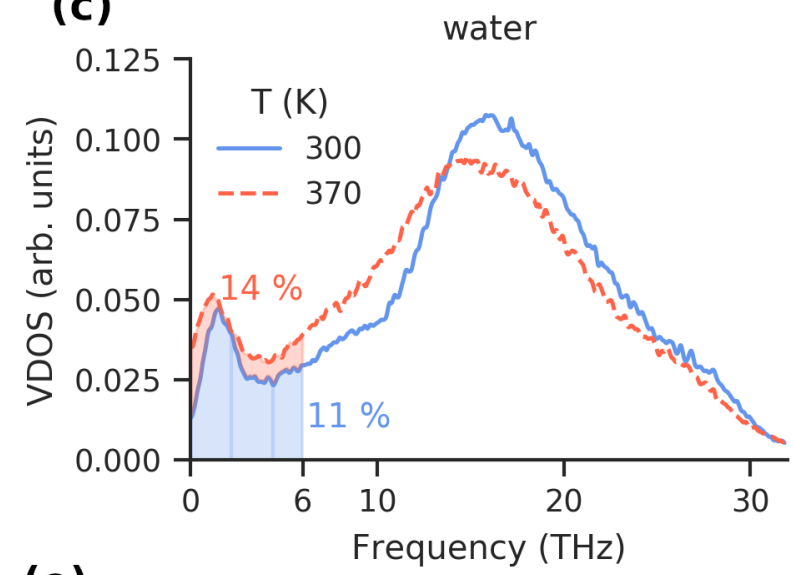

(e)

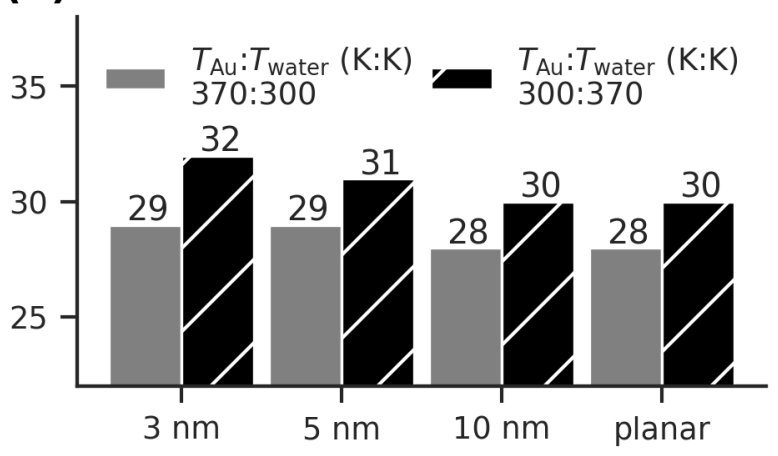

Figure 7. Analysis of the vibrational density of states (VDOS). a) VDOS of gold and water at $300 \mathrm{~K}$. The grey region denotes the frequency range 0 to $6 \mathrm{THz}$ which is shown in more detail in the inset. b) VDOS of the $3 \mathrm{~nm}$ AuNP at $300 \mathrm{~K}$ and $370 \mathrm{~K} \mathrm{c}$ ) Comparison of the water VDOS at $300 \mathrm{~K}$ and $370 \mathrm{~K}$. The shaded areas highlight the density in the range 0 to $6 \mathrm{THz}$ with the labeled percentage by area. Note that in each case when plotting the VDOS we re-scaled the density by the maximum value of the $10 \mathrm{~nm}$ AuNP so that y-axis ranges from 0 to $\sim 1$. d) Comparison of the $B C$ values for each gold surface computed from the VDOS at $300 \mathrm{~K}$ and $370 \mathrm{~K}$ (gold and water are at the same temperature in each case). e) Cross-temperature $B C$ values for the two combinations of gold and water at different temperature $(300 \mathrm{~K}$ or $370 \mathrm{~K})$. 


\section{Methods}

\subsection{Model System Construction}

We constructed four atomistic models of nanoscale-gold immersed in water: three spherical AuNPs with diameters of 3,5 , and $10 \mathrm{~nm}$, as well as a slab of gold with a planar gold-water interface. The nanoscale-gold structures were constructed and solvated using VMD software. 67. The planar gold slab was constructed as a rectangular block of $\mathrm{Au}_{111}$ with dimensions of $69.4 \times 69.4 \times 70.4 \AA^{3}$ (20230 gold atoms) and the two faces along the z-dimension were solvated with layers of water with thickness $190.7 \AA$ (185919 total water atoms including both layers). Overall, the planar-gold water system consisted of a total of 206149 atoms with box dimensions of $69.4 \times 69.4 \times 450.8 \AA^{3}$ with the gold slab centered at the origin. Each AuNP was constructed by extracting a spherical selection of atoms within a radius equal to that of the AuNP's nominal radius from a large block of Au ${ }_{111}$. The AuNPs consisted of 887, 3925, and 30885 gold atoms for the 3,5, and $10 \mathrm{~nm}$ AuNPs, respectively. The AuNPs were then solvated with cubic boxes of water containing 88317,187459 , and 378583 water molecules with side lengths of $140.00,180.00$, and $230.00 \AA$ for the 3, 5, and $10 \mathrm{~nm}$ AuNPs, respectively. In all AuNP systems, the AuNP was centered at the origin.

\subsection{Non-equilibrium steady-state molecular dynamics simulations}

All molecular dynamics simulations were carried out with the LAMMPS software (http://lammps.sandia.gov) using periodic boundary conditions and a timestep of $1 \mathrm{fs} .68$ Note that under periodic boundary conditions, the planar gold slab extends semi-infinitely in the xy plane.

We modeled nanoscale-gold using the embedded atom model, 69 which is a many-body potential that more accurately represents metallic bonding than standard pairwise potentials. Water was modeled using the flexible SPCE-F model, 70 chosen primarily for its ability to reasonably estimate the experimental surface tension of water at temperatures between 300-500 K. With the SPCE-F model, the van der Waals interactions are described by a standard 12-6 Lennard-Jones potential, defined as

$$
U_{i j}=4 \epsilon_{i j}\left[\left(\frac{\sigma_{i j}}{r}\right)^{12}-\left(\frac{\sigma_{i j}}{r}\right)^{6}\right], r<r_{c}
$$

for atoms i and $\mathrm{j}$ separated by distance $r$, with a cutoff distance of $r_{c} . \epsilon_{i j}$ is the energy coefficient $\left(\mathrm{kJ} \mathrm{mol}{ }^{-1}\right.$ ) describing the strength of the interaction, and $\sigma_{i j}(\AA)$ is the zero-energy distance describing the sizes of interacting atoms. Only the water O-O interactions are non-zero with $\epsilon_{O-O}=0.65 \mathrm{~kJ} \mathrm{~mol}^{-1}$ and $\sigma_{O-O}=3.1661 \AA$. The intramolecular $\mathrm{H}-\mathrm{O}$ bond stretching is modeled with a harmonic potential

$$
U_{\text {bond }}=K_{b}\left(b-b_{0}\right)^{2},
$$

for bond distance $b$ with force constant $K_{b}=2322.20 \mathrm{~kJ} \mathrm{~mol}^{-1} \AA^{-2}$ and equilibrium bond distance $b_{0}=1.0 \AA$. The intramolecular $\mathrm{H}-\mathrm{O}-\mathrm{H}$ angle is also maintained with a harmonic potential

$$
U_{\text {angle }}=K_{a}\left(\theta-\theta_{0}\right)^{2},
$$

for angle $\theta$ with force constant $K_{a}=191.7549 \mathrm{~kJ} \mathrm{~mol}^{-1} \mathrm{rad}^{-2}$ and equilibrium angle $\theta_{0}=109.4^{\circ}$. The interaction between gold and water was also modeled using a standard 12-6 Lennard-Jones potential. Only the oxygen-gold interactions were included in the description of the gold-water interaction. In the moderate wetting case, the parameters for the water oxygen and gold interaction were $\epsilon_{O-A u}=2.47 \mathrm{~kJ} \mathrm{~mol}^{-1}$ and $\sigma_{O-A u}=3.6 \AA$ as developed by Merabia et al. 35 to reproduce a moderate wetting condition with a gold-water contact angle $<30^{\circ}$. In the poor wetting case, the parameters were adjusted to $\epsilon_{O-A u}=0.59 \mathrm{~kJ} \mathrm{~mol}^{-1}$ and $\sigma_{O-A u}=3.383 \AA$, yielding a weaker gold-water interaction. The model parameters are summarized in Table S4 (Supporting Information). A global cutoff of $r_{c}=10 \AA$ was used for the Lennard-Jones interactions. The particle-particle particle-mesh solver was used for electrostatics with a desired relative error in the forces of 0.0001 with a switching cutoff of $8 \AA$ for smoothly transitioning to long range electrostatics. 71 The gold structures were prevented from drifting during the simulations by tethering their center of mass to the origin using a harmonic spring with force constant $960 \mathrm{~kJ} \mathrm{~mol}^{-2} \AA^{-1}$. 
Initially, each nanoscale gold-water system was energy minimized and then equilibrated under NVT conditions for 1 ns at $300 \mathrm{~K}$, followed by an additional equilibration phase under NPT conditions for $1 \mathrm{~ns}$ at $300 \mathrm{~K}$ and 1 atm. The non-equilibrium steady-state simulations were carried out under NPH conditions at 1 atm. The gold structure was subjected to continuous heating at a given heat power $Q$, distributed uniformly across the gold atoms. In order to create a non-equilibrium heat flux an outer region of water was maintained at $300 \mathrm{~K}$ with a Langevin thermostat, 72 serving as a heat sink. In the planar gold-water system, the heat sink was defined as the region encompassing $z>200$ $\AA$ and $z<-200 \AA$ within the unit cell; note that these two regions are joined into a single region by the periodic boundary conditions. In the AuNP systems, the heat sink region was defined using a radial distance criterion, namely the region encompassing $r>65 \AA, r>85 \AA$, and $r>110 \AA$ from the origin, for the 3,5, and $10 \mathrm{~nm}$ AuNPs, respectively. The non-equilibrium steady-state simulations were run for $2,3,4$ and $6 \mathrm{~ns}$, for the 3,5 , and $10 \mathrm{~nm}$ AuNPs and planar slab, respectively. For the poor wetting case, the simulation times were all 4 ns.

\subsection{Analysis}

Simulation analysis was carried out using a combination of LAMMPS functionality, Python code (https://www.python.org), and VMD scripts (https://www.ks.uiuc.edu/Research/vmd). 67. Our analysis in Python incorporated various libraries, including MDanalysis, 73 74. SciPy, 75 NumPy, 76 Matplotlib, 77 seaborn, 78 Numba, 79 pandas, $[80$ and Jupyter. 81 Simulation snapshots were rendered from VMD using the Tachyon ray tracer. 82

In the analysis of the non-equilibrium steady state simulations, we discarded the first 1 ns of the AuNP system trajectories and the first $3 \mathrm{~ns}$ of the planar slab system. In the poor wetting case, we adjusted the time to 2 ns for the 5 and $10 \mathrm{~nm}$ AuNP systems. We visually inspected the time course of the gold temperature (Figure S8 and Figure S9, Supporting Information) to confirm that the systems were no longer in the transient phase and had reached a steady-state by those times. Descriptions of individual analyses are provided in subsequent sections.

\subsubsection{Temperature profiles}

Instantaneous spatial temperature profiles for gold and water were computed separately with a spatial binning of width $\sim 1 \AA$ (radial shells for AuNPs and rectangular blocks along the z-direction for the planar slab) every 1 ps during non-equilibrium steady state simulations. Subsequently, bins with $<100$ atoms at any time point were dropped from the profile to reduce errors in the temperature estimation. The steady-state temperature profiles were then estimated by time averaging the instantaneous profiles within the designated steady-state portions of each trajectory.

\subsubsection{Temperature jump and thermal interface conductance}

The average values and standard errors of the thermal interface conductance $G$ values were estimated by block averaging of the temperature profile. 83 For each block, $\Delta T$ was estimated as the temperature difference between the outermost gold spatial bin and the innermost water spatial bin. To account for the possibility of thermal expansion of the gold, the gold size (diameter for AuNPs and thickness for planar slab) was estimated from the outermost gold spatial bin position (Table S2, Supporting Information). The corresponding $G$ for the block was estimated according to Equation 1. Block sizes of 200 ps, 250 ps, 500 ps, and 750 ps were used for the block averages for the 3, 5, and 10 nm AuNPs and planar slab, respectively. In the poor wetting case, the block sizes were adjusted to 200 ps, 250 ps, $400 \mathrm{ps}$, and $300 \mathrm{ps}$ for the 3, 5, and $10 \mathrm{~nm}$ AuNPs and planar slab, respectively. The gold temperature was examined using blocked standard error analysis with varying block sizes (Figure S10 and Figure S11, Supporting Information) to confirm that these block sizes resulted in uncorrelated data for block averaging. The reported error in $G$ values was taken as 1.96 times the standard error estimate (from block averaging) and thus represents an estimate of the $95 \%$ confidence interval.

\subsubsection{Mass density profiles}

Instantaneous (spatial) mass density profiles for gold and water were computed separately with a spatial binning of width $0.25 \AA$ (radial shells for AuNPs and rectangular blocks along the z-direction for the planar surface). At each 
time step, the coordinates of atoms were shifted so that the center of mass of the gold structure was at the origin. In the case of the planar surface, the profile was averaged over the positive and negative z-directions. The profiles for systems in the absence of heating were computed by averaging over the final 500 ps of the equilibration simulations, which were under NPT conditions at $300 \mathrm{~K}$ and 1 atm. For systems under heating, the steady-state profile was computed by time averaging the instantaneous profiles over the final 1 ns of each non-equilibrium steady-state simulation. The time averaged profiles were then further smoothed by applying a Gaussian filter with $\sigma=2$ to generate the final equilibrium or steady-state profile.

\subsubsection{Surface Adsorption Density}

The surface adsorption density $(\varrho)$ was computed according to the following equations adapted from Tascini et al.: 27. for spherical AuNPs,

and the planar gold surface,

$$
\varrho=\frac{N_{w}}{A}=\frac{4 N_{A}}{M_{w} d^{2}} \int_{0}^{r_{\max }} r^{2} \rho_{w}(r) d r
$$

$$
\varrho=\frac{N_{w}}{A}=\frac{N_{A}}{M_{w}} \int_{0}^{z_{\max }} \rho_{w}(z) d z,
$$

where $N_{w}$ is the number of water molecules adsorbed on the surface of gold with surface area $A, N_{A}$ is Avogadro's number, $M_{w}$ the molar mass of water, $\rho_{w}(r)$ is the radial mass density of water at radius $r$, and $\rho_{w}(z)$ is the mass density of water along the direction perpendicular to the planar surface, $z$. The upper limits of the integrals, $r_{m a x}$ and $z_{\max }$, were taken as the profile position corresponding to the minimum following the peak of the adsorption band (for example, see Figure 5b,c). The integrals were estimated using numerical quadrature.

\subsubsection{Solvent-accessible surface area}

Solvent-accessible surface area estimates were computed using the measure sasa function in VMD with a probe radius of $4.04 \AA$, 67. which corresponds to the minimum energy in the Lennard-Jones potential for the gold-water interaction in the moderate wetting case.

\subsubsection{Vibrational density of states}

The vibrational density of states (VDOS) were estimated by taking a Fourier transform of the velocity autocorrelation function, as given by the following equation:

$$
\operatorname{VDOS}(\omega)=\int_{0}^{+\infty}\langle\vec{v}(t) \cdot \vec{v}(0)\rangle e^{-i \omega t} d t,
$$

where $\omega$ is the vibrational frequency and $\langle\vec{v}(t) \cdot \vec{v}(0)\rangle$ is the velocity autocorrelation function computed and averaged over all atoms of given type (either gold or water) and over multiple time origins (denoted by angular brackets). The velocity autocorrelation function was computed by a separate set of simulations executed with a time step of 0.25 fs and NVE conditions, initialized from the final configurations of each system after equilibration under NPT conditions at the specified temperature $(300$ or $370 \mathrm{~K})$ and 1 atm. The simulations were executed for a total of 40000 time steps, and the velocity autocorrelation function was computed for two time origins taken over two consecutive 20000 time step intervals.

\subsubsection{Bhattacharyya coefficient for overlap between gold and water vibrational density of states}

The Bhattacharyya coefficient (BC) quantifying the level of overlap between the gold and water vibrational density of states was computed using numerical estimation of the following integral: 66

$$
B C=\int \sqrt{\operatorname{VDOS}_{A u}(\omega) \times \operatorname{VDOS}_{\text {Water }}(\omega)} d \omega,
$$

where $\omega$ is the vibrational frequency and $\operatorname{VDOS}_{A u}(\omega)$ and $\operatorname{VDOS}_{W a t e r}(\omega)$ are the vibrational density of states (VDOS) of gold and water, respectively. The integral was taken on the entire range of frequencies over which the VDOS was computed (0-32 THz). 


\section{Supporting Information}

Supporting Information is available online or from the author.

\section{Acknowledgements}

The authors thank Dr. Hejian Xiong, Dr. Haihang Ye, Dr. Qi Cai, and Chen Xie for critically reading the manuscript and providing their feedback. Research reported in this work was supported by the National Institutes of Health under award number R35GM133653. The authors also acknowledge the Texas Advanced Computing Center (TACC) at The University of Texas at Austin for providing HPC resources that have contributed to these research results. URL: http://www.tacc.utexas.edu

\section{Conflict of Interest}

The authors declare no conflict of interest.

\section{References}

1. G. Baffou, R. Quidant, Laser and Photonics Reviews 2013, 7, 2171.

2. Z. Qin, J. C. Bischof, Chemical Society Reviews 2012, 41, 31191.

3. G. Baffou, F. Cichos, R. Quidant, Nature Materials 2020, 19, September.

4. L. R. Hirsch, R. J. Stafford, J. A. Bankson, S. R. Sershen, B. Rivera, R. E. Price, J. D. Hazle, N. J. Halas, J. L. 385 West, Proceedings of the National Academy of Sciences of the United States of America 2003, 100, 2313549.386

5. G. S. Terentyuk, G. N. Maslyakova, L. V. Suleymanova, N. G. Khlebtsov, B. N. Khlebtsov, G. G. Akchurin, ${ }^{387}$ I. L. Maksimova, V. V. Tuchin, Journal of Biomedical Optics 2009, 14, 2021016.

6. G. Huettmann, J. Serbin, B. Radt, B. I. Lange, R. Birngruber, Laser-Tissue Interaction XII: Photochemical, 389 Photothermal, and Photomechanical 2001, 425\%, July 2001398.

7. G. Huettmann, B. Radt, J. Serbin, R. Birngruber, G. Huttmann, B. Radt, J. Serbin, R. Birngruber, In R. W. 391 Steiner, editor, Proc. SPIE, volume 5142. International Society for Optics and Photonics, 200388.

8. C. M. Pitsillides, E. K. Joe, X. Wei, R. R. Anderson, C. P. Lin, Biophysical Journal 2003, 84, 64023.

9. P. Kang, Z. Chen, S. O. Nielsen, K. Hoyt, S. D’Arcy, J. J. Gassensmith, Z. Qin, Small 2017, 13, 361700841.394

10. P. Kang, X. Li, Y. Liu, S. I. Shiers, H. Xiong, M. Giannotta, E. Dejana, T. J. Price, J. Randrianalisoa, S. O. Nielsen, Z. Qin, ACS Nano 2019, 1312487.

11. K. Metwally, S. Mensah, G. Baffou, Journal of Physical Chemistry C 2015, 119, 5128586.

12. A. Carattino, M. Caldarola, M. Orrit, Nano Letters 2018, 18, 2874.

13. D. A. Hastman, S. A. Diaz, ACS Nano 2020.

14. D. Boyer, P. Tamarat, A. Maali, B. Lounis, M. Orrit, Science 2002, 297, 55841160.

15. M. Selmke, M. Braun, F. Cichos, ACS Nano 2012, 6, 32741.

16. Y. S. Chen, W. Frey, S. Kim, P. Kruizinga, K. Homan, S. Emelianov, Nano Letters 2011, 11, 2348.

17. X. Liu, M. G. González, R. Niessner, C. Haisch, Analytical Methods 2012, 4, 1309. 
18. D. G. Cahill, W. K. Ford, K. E. Goodson, G. D. Mahan, A. Majumdar, H. J. Maris, R. Merlin, S. R. Phillpot, ${ }^{404}$ Journal of Applied Physics 2003, 93, 2793.

19. T. Luo, G. Chen, Physical Chemistry Chemical Physics 2013, 15, 103389.

20. O. M. Wilson, X. Hu, D. G. Cahill, P. V. Braun, Physical Review B - Condensed Matter and Materials Physics 2002, 66, 222243011.

21. A. Plech, V. Kotaidis, S. Grésillon, C. Dahmen, G. von Plessen, Physical Review B 2004, $70,19195423$.

22. Z. Ge, D. G. Cahill, P. V. Braun, Journal of Physical Chemistry B 2004, 108, 4918870.

23. Z. Ge, D. G. Cahill, P. V. Braun, Physical Review Letters 2006, 96, 18186101.

24. A. J. Schmidt, J. D. Alper, M. Chiesa, G. Chen, S. K. Das, K. Hamad-Schifferli, Journal of Physical Chemistry $C$ 2008, 112, 3513320.

25. J. Alper, K. Hamad-Schifferli, Langmuir 2010, 26, 63786.

26. T. Stoll, P. Maioli, A. Crut, S. Rodal-Cedeira, I. Pastoriza-Santos, F. Vallée, N. Del Fatti, Journal of Physical Chemistry $C$ 2015, 119, 2212757.

27. A. S. Tascini, J. Armstrong, E. Chiavazzo, M. Fasano, P. Asinari, F. Bresme, Physical Chemistry Chemical Physics 2017, 19, 43244.

28. A. R. Nair, S. P. Sathian, Journal of Heat Transfer 2016, 138, 111.

29. A. Lervik, F. Bresme, S. Kjelstrup, Soft Matter 2009, 5, 122407.

30. K. M. Stocker, S. M. Neidhart, J. D. Gezelter, Journal of Applied Physics 2016, $119,2025106$.

31. S. M. Neidhart, J. D. Gezelter, Journal of Physical Chemistry C 2018, 122, 21430.

32. M. Hu, G. V. Hartland, Journal of Physical Chemistry B 2002, 106, 287029.

33. X. Wei, T. Zhang, T. Luo, ACS Energy Letters 2017, 2, 102283.

34. Y. Wang, P. Keblinski, Applied Physics Letters 2011, 99, 7.

35. S. Merabia, S. Shenogin, L. Joly, P. Keblinski, J.-L. Barrat, Proceedings of the National Academy of Sciences of the United States of America 2009, 106, 3615113.

36. Y. Dou, L. V. Zhigilei, N. Winograd, B. J. Garrison, Journal of Physical Chemistry A 2001, $105,122748$.

37. P. Schravendijk, N. Van Der Vegt, L. Delle Site, K. Kremer, ChemPhysChem 2005, 6, 91866.

38. K. A. Tay, F. Bresme, Journal of the American Chemical Society 2006, 128, 4314166.

39. A. V. Verde, J. M. Acres, J. K. Maranas, Biomacromolecules 2009, 10, 82118.

40. X. Chen, A. Munjiza, K. Zhang, D. Wen, The Journal of Physical Chemistry C 2014, $118,21285$.

41. F. Iori, S. Corni, Journal of Computational Chemistry 2008, 29, 101655.

42. F. Iori, R. Di Felice, E. Molinari, S. Corni, Journal of Computational Chemistry 2008, 30, 91465.

43. H. Bhattarai, K. E. Newman, J. D. Gezelter, Journal of Chemical Physics 2020, $153,20$.

44. J. V. Goicochea, M. Hu, B. Michel, D. Poulikakos, Journal of Heat Transfer 2011, $133,83$.

45. S. W. Hung, G. Kikugawa, J. Shiomi, Journal of Physical Chemistry C 2016, 120, 4726678. 
46. K. M. Stocker, J. D. Gezelter, The Journal of Physical Chemistry C 2013, 117, 157605.

47. S. Salassi, A. Cardellini, P. Asinari, R. Ferrando, G. Rossi, Nanoscale Advances 2020.

48. A. Plech, S. Ibrahimkutty, S. Reich, G. Newby, Nanoscale 2017, 9, 4417284.

49. C. W. Nan, R. Birringer, D. R. Clarke, H. Gleiter, Journal of Applied Physics 1997, 81, 106692.

50. C. W. Nan, R. Birringer, Physical Review B - Condensed Matter and Materials Physics 1998, 57, 148264.

51. H. K. Lyeo, D. G. Cahill, Physical Review B - Condensed Matter and Materials Physics 2006, 73, 141.

52. P. E. Hopkins, P. M. Norris, R. J. Stevens, Journal of Heat Transfer 2008, 130, 2.

53. S. Murad, I. K. Puri, Applied Physics Letters 2008, 92, 1310.

54. S. Murad, I. K. Puri, Chemical Physics Letters 2008, 467, 1-3 110.

55. G. Balasubramanian, S. Banerjee, I. K. Puri, Journal of Applied Physics 2008, 104, 6.

56. B. H. Kim, A. Beskok, T. Cagin, Journal of Chemical Physics 2008, 129, 17.

57. Z. Shi, M. Barisik, A. Beskok, International Journal of Thermal Sciences 2012, 5929.

58. M. Hu, D. Poulikakos, C. P. Grigoropoulos, H. Pan, Journal of Chemical Physics 2010, $132,16$.

59. N. Shenogina, R. Godawat, P. Keblinski, S. Garde, Physical Review Letters 2009, 102, 15156101.

60. B. Lee, F. M. Richards, Journal of Molecular Biology 1971, 55, 3.

61. A. Shrake, J. A. Rupley, Journal of Molecular Biology 1973, 79, 2361.

62. E. T. Swartz, R. O. Pohl, Reviews of Modern Physics 1989, 61, 3605.

63. P. Reddy, K. Castelino, A. Majumdar, Applied Physics Letters 2005, 87, 211.

64. J. W. Lynn, H. G. Smith, R. M. Nicklow, Physical Review B 1973, 8, 83493.

65. R. Carles, P. Benzo, B. Pécassou, C. Bonafos, Scientific Reports 2016, 6, 11.

66. A. Bhattacharya, The Indian Journal of Statistics 1946, 7, 4401.

67. W. Humphrey, A. Dalke, K. Schulten, Journal of Molecular Graphics 1996, 1433.

68. S. Plimpton, Journal of computational physics 1995, 117, 11.

69. S. M. Foiles, M. I. Baskes, M. S. Daw, Physical Review B 1986, 33, 127983.

70. J. López-Lemus, G. A. Chapela, J. Alejandre, Journal of Chemical Physics 2008, 128, 17.

71. R. Hockney, J. Eastwood, Computer Simulation Using Particles 1989, 120-165.

72. T. Schneider, E. Stoll, Physical Review B 1978, 17, 31302.

73. N. Michaud-Agrawal, E. J. Denning, T. B. Woolf, O. Beckstein, Journal of computational chemistry 2011, 32, 465 102319.

74. Richard J. Gowers, Max Linke, Jonathan Barnoud, Tyler J. E. Reddy, Manuel N. Melo, Sean L. Seyler, Jan Domański, David L. Dotson, Sébastien Buchoux, Ian M. Kenney, Oliver Beckstein, In Sebastian Benthall, Scott Rostrup, editors, Proceedings of the 15th Python in Science Conference. 201698 - 105. 
75. P. Virtanen, R. Gommers, T. E. Oliphant, M. Haberland, T. Reddy, D. Cournapeau, E. Burovski, P. Peterson, ${ }_{470}$ W. Weckesser, J. Bright, S. J. van der Walt, M. Brett, J. Wilson, K. J. Millman, N. Mayorov, A. R. J. Nelson, ${ }^{471}$ E. Jones, R. Kern, E. Larson, C. J. Carey, I. Polat, Y. Feng, E. W. Moore, J. VanderPlas, D. Laxalde, J. Perktold, R. Cimrman, I. Henriksen, E. A. Quintero, C. R. Harris, A. M. Archibald, A. H. Ribeiro, F. Pedregosa, P. van Mulbregt, SciPy 1.0 Contributors, Nature Methods 2020, 17261.

76. C. R. Harris, K. J. Millman, S. J. van der Walt, R. Gommers, P. Virtanen, D. Cournapeau, E. Wieser, J. Taylor, S. Berg, N. J. Smith, R. Kern, M. Picus, S. Hoyer, M. H. van Kerkwijk, M. Brett, A. Haldane, J. Fernández del Río, M. Wiebe, P. Peterson, P. Gérard-Marchant, K. Sheppard, T. Reddy, W. Weckesser, H. Abbasi, C. Gohlke, T. E. Oliphant, Nature 2020, 585357.

77. J. D. Hunter, Computing in Science Engineering 2007, 9, 390.

78. M. L. Waskom, Journal of Open Source Software 2021, 6, 603021.

79. S. K. Lam, A. Pitrou, S. Seibert, In Proceedings of the Second Workshop on the LLVM Compiler Infrastructure in HPC, LLVM '15. Association for Computing Machinery, New York, NY, USA, 2015.

80. Wes McKinney, In Proceedings of the 9th Python in Science Conference. 201056 - 61.

81. T. Kluyver, B. Ragan-Kelley, F. Pérez, B. Granger, M. Bussonnier, J. Frederic, K. Kelley, J. Hamrick, J. Grout, S. Corlay, P. Ivanov, D. Avila, S. Abdalla, C. Willing, In F. Loizides, B. Schmidt, editors, Positioning and Power in Academic Publishing: Players, Agents and Agendas. IOS Press, 201687 - 90.

82. J. Stone, Master's thesis, Computer Science Department, University of Missouri-Rolla, 1998.

83. A. Grossfield, D. M. Zuckerman, In Annual Reports in Computational Chemistry, volume 5, chapter 2, 23 - 48.488 Elsevier, 2009. 